\title{
Características técnico-económicas de sistemas de producción bovina de las razas criollas colombianas Romosinuano y Hartón del Valle
}

\author{
Rosa Parra-Cortés ${ }^{凶}$ M.Sc; Miguel Magaña-Magaña ${ }^{1 *} \otimes(\mathbb{0}$ Ph.D.
}

${ }^{1}$ Tecnológico Nacional de México/IT Conkal, División de Estudios de Posgrado e Investigación. Avenida Tecnológico s/n, C.P. 97345, Conkal, Yucatán, México.

*Correspondencia: drmmagana@gmail.com

Recibido: Junio 2020; Aceptado: Marzo 2021; Publicado: Abril 2021.

\section{RESUMEN}

Objetivo. Identificar y caracterizar los sistemas de producción bovina basados en razas criollas Romosinuano y Hartón del Valle en condiciones de trópico bajo colombiano. Materiales y métodos. Se realizó una encuesta por muestreo aleatorio a productores en los departamentos de Antioquia, Bolívar, Boyacá, Córdoba, Cesar, Cundinamarca, Meta, Santander, Sucre y Valle del Cauca. Se determinaron las principales características socioeconómicas de los productores y se clasificaron los sistemas de producción según su finalidad; asimismo, se establecieron su respectivos niveles tecnológicos e indicadores de desempeño técnico-económico. Resultados. La edad promedio de los productores fue de 58 años, con escolaridad de nivel superior y experiencia en la actividad ganadera de 20 años. Los principales parámetros de desempeño animal estimados para la raza Romosinuano fueron peso al nacimiento $28.0 \pm 1.3 \mathrm{~kg}$, peso al destete $185.04 \pm 45.5 \mathrm{~kg}$, tasa de fertilidad $80 \pm 16.0 \%$ e intervalo entre partos de $408 \pm 43.0$ días. Asimismo, para la raza Hartón del Valle que los citados parámetros fueron $28.1 \pm 0.32 \mathrm{~kg}, 199.5 \pm 8.3 \mathrm{~kg}, 82 \pm 9.0 \%$ y $354 \pm 13.0$ días, respectivamente. El nivel tecnológico de los sistemas fue categorizado como medio-alto y su desempeño económico evidenció un índice de rentabilidad anual superior al 12\%. Conclusiones. Los sistemas de producción basados en razas criollas en el trópico bajo colombiano, se caracterizaron, principalmente por un nivel tecnológico relevante y parámetros diferenciados entre razas. La rentabilidad se vinculó tanto al manejo técnico como a las condiciones socioeconómicas del productor.

Palabras clave: Desempeño animal; ganadería; productividad; recursos genéticos animales; sistemas de producción; rentabilidad (Fuente: AGROVOC Multilingual Thesaurus).

\section{ABSTRACT}

Objective. Identify and characterize bovine production systems based on Romosinuano and Hartón del Valle creole breeds in lowland colombian tropical conditions. Materials and methods. A random sampling survey was conducted among producers in the departments of Antioquia, Bolívar, Boyacá, Córdoba, Cesar, Cundinamarca, Meta, Santander, Sucre and Valle del Cauca. The main socioeconomic characteristics of the producers were determined and the production systems were

Como citar (Vancouver).

Parra-Cortés R, Magaña-Magaña M. Características técnico-económicas de sistemas de producción bovina de las razas criollas colombianas Romosinuano y Hartón del Valle. Rev MVZ Córdoba. 2021; 26(2):e2079. https://doi.org/10.21897/rmvz.2079 
classified according to their purpose; likewise, their respective technological levels and technicaleconomic performance indicators were established. Results. The average age of the producers was 58 years old, with a higher level of education and 20 years of experience in livestock farming. The main animal performance parameters estimated for the Romosinuano breed were birth weight $28.0 \pm 1.3 \mathrm{~kg}$, weaning weight $185.04 \pm 45.5 \mathrm{~kg}$, fertility rate $80 \pm 16.0 \%$ and calving interval of $408 \pm 43.0$ days. Likewise, for the Hartón del Valle breed, the mentioned parameters were $28.1 \pm 0.32$ $\mathrm{kg}, 199.5 \pm 8.3 \mathrm{~kg}, 82 \pm 9.0 \%$ and $354 \pm 13.0$ days, respectively. The technological level of the systems was categorized as medium-high and their economic performance showed an annual profitability index of over $12 \%$. Conclusions. The production systems based on creole breeds in the lowland colombian tropics were characterized, mainly by a relevant technological level and differentiated parameters between breeds. The profitability was linked to both technical management and the producer's socioeconomic conditions.

Keywords: Animal performance; livestock; productivity; animal genetic resources; production systems; profitability (Source: AGROVOC Multilingual Thesaurus).

\section{INTRODUCCIÓN}

La demanda mundial se observa que la demanda de productos de origen animal para la alimentación humana es creciente. Se estima que, para el año 2050, el consumo de carnes proveniente de rumiantes supere en $200 \%$ las cifras actuales. Las causas que originan estos incrementos difieren entre países desarrollados y aquellos en vías de desarrollo; en los primeros se destaca el aumento en los ingresos familiares de la clase media, así como el cambio en el patrón de preferencias de los consumidores y la migración humana hacia las ciudades o polos de desarrollo. En los países en desarrollo la principal causa del citado incremento se asocia al crecimiento demográfico; sin embargo, una mayor demanda de alimentos no se refleja en el dinamismo económico de las regiones rurales, por lo tanto, existen preocupaciones relacionadas con la pobreza y seguridad alimentaria.

La demanda de alimentos ha provocado cambios en la economía global, que otorgan a los agroecosistemas mayores exigencias técnicoproductivas, lo que da como resultado, entre otros aspectos relevantes, la intensificación de la ganadería en diversas partes del mundo; condición que se relaciona con incrementos en la capacidad de carga animal por unidad de superficie, el uso de piensos y la mejora del mérito genético de los ganados (1). En la actualidad, los sistemas ganaderos no solo deben garantizar los incrementos en la producción, sino que además se enfrentan a los retos derivados del cambio climático, tales como la aparición de enfermedades, estrés por calor, escasez o exceso de agua, baja cantidad y calidad de forrajes y reducción de la biodiversidad $(2,3)$.
El fenómeno de intensificación ganadera ha provocado el uso intensivo de un menor número de razas consideradas altamente productivas $y$, a su vez, una subutilización de los recursos zoogenéticos locales (4). Al respecto, se conoce que la población de bovinos criollos en América Latina (animales de origen europeo que evolucionaron y se adaptaron en el continente americano desde tiempos de la conquista), se ha reducido drásticamente desde finales del siglo XIX. En la actualidad, en la mayoría de países, se desconocen los inventarios de estos animales y se dispone de escasa información sobre su aporte tanto a la economía como a los medios de vida de las comunidades rurales. Sin embargo, existen evidencias sobre la destacada fertilidad de las razas criollas, su longevidad, mansedumbre, resistencia a parásitos y tolerancia al estrés calórico, así como la sobresaliente calidad organoléptica y nutricional de su carne y leche (5).

Las anteriores características zootécnicas de los bovinos criollos los posicionan como una fuente de diversidad genética, útil tanto en estado puro como en planes de cruzamientos con razas comerciales; ya que su acervo génico podría conferir a su descendencia una mejor capacidad de adaptación al entorno y a los riesgos derivados del cambio climático. Entre los países latinoamericanos con mayor número de razas bovinas criollas se encuentra Colombia, el cual cuenta con once tipos raciales (Arhuaco, Blanco orejinegro, Campuzano, Casanare, Caqueteño, Chino santandereano, Costeño, Hartón del Valle, Lucerna, Romosinuano, Sanmartinero y Velásquez) y un inventario precario estimado en $1 \%$ del censo bovino nacional (6). En general, la actividad ganadera en este país cumple un rol importante en la economía nacional, posee 
un inventario de 27.234.027 cabezas bovinas distribuidas en 623.794 predios (7); entre los principales departamentos productores destacan Antioquia (11.35\%), Córdoba $(7.84 \%)$, Casanare $(7.84 \%)$ y Meta $(7.51 \%)$.

En países de América Latina, como en Colombia, aunque existen algunos antecedentes (8), no están documentadas recientemente las características técnicas, la importancia económica y las perspectivas de los sistemas de producción ganaderos basados en razas criollas. Entre las razas bovinas criollas más difundidas en el trópico colombiano se encuentran la romosinuano (también conocida como Romo) y la Hartón del Valle. La primera es de finalidad cárnica y recibe su nombre a la ausencia de cuernos y su lugar de origen es el valle del río Sinú, Departamento de Córdoba. Por su parte, la Hartón del Valle es considerada una raza de doble propósito, tiene su asentamiento en el valle del rio del Cauca. En el presente, estas razas se encuentran en diversas regiones de Colombia y se caracterizan por poseer un tamaño pequeño a medio, con capa de pelaje de color amarillo claro a rojo encendido, así como es posible encontrar animales hoscos, es decir, con cabeza y extremidades negras (9). Por lo tanto, el objetivo de este trabajo fue identificar y caracterizar los principales atributos técnico-económicos de los sistemas de producción bovina basados en ejemplares de las razas criollas Romosinuano y Hartón del Valle, en zonas con condiciones agroclimáticas de trópico bajo colombiano.

\section{MATERIALES Y MÉTODOS}

Localización y descripción del área de estudio. Se consideraron unidades de producción ubicadas en altitudes de 0 hasta 1.000 msnm (Trópico bajo), pertenecientes a la región andina en los departamentos de Antioquia, Boyacá, Cundinamarca, Valle del Cauca y Santander; a la Orinoquía en Meta; así como, a la región Caribe en Bolívar, Córdoba, Cesar y Sucre (Figura 1). Las regiones seleccionadas incluyeron zonas con climas cálido seco con un rango de precipitación anual de 1.001 a 2.000 mm, así como cálido húmedo con 2.001 a $3.000 \mathrm{~mm}$ y una temperatura promedio anual de $24^{\circ} \mathrm{C}$ (10)

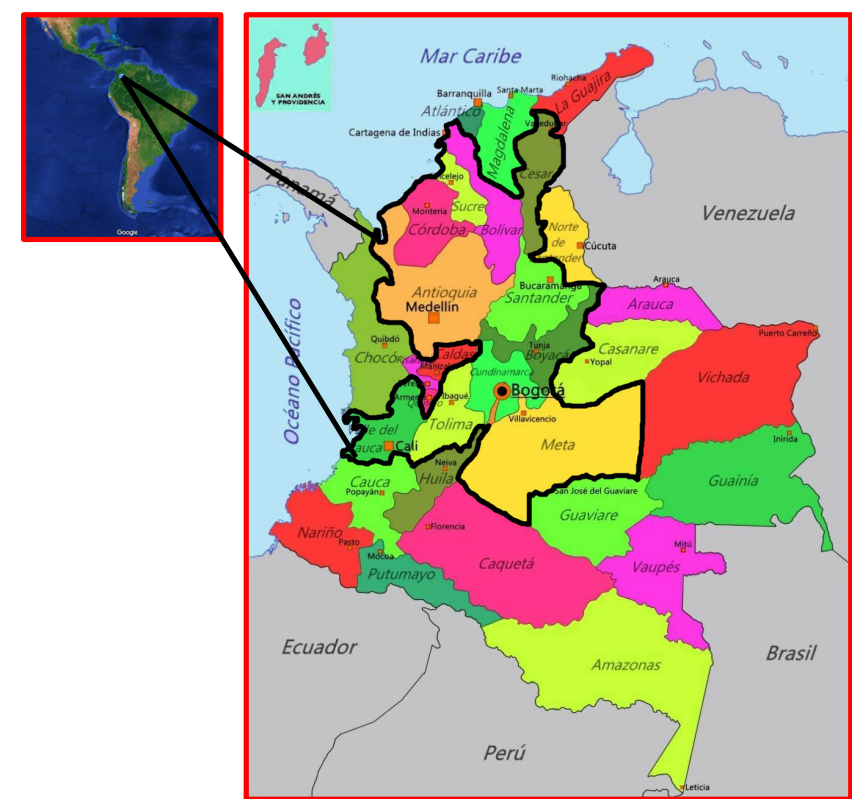

Fuente: Adaptado de IGAC y Google Maps, 2019

Figura 1. Ubicación del país y localización del área de estudio

Universo de estudio. Se consideró a los productores vinculados con la Asociación Nacional de Criadores de Razas Criollas y Colombianas (ASOCRIOLLO) y la Asociación de Criadores de Razas Criollas y Colombianas de Los Llanos Orientales (ASOCRIOLLANOS). Se solicitó a la directiva de cada organización el listado de productores que crían las razas Romosinuano y Hartón del Valle. Posteriormente, se conformó un listado con 49 productores localizados en el área de estudio.

Obtención de la información. La información de interés se obtuvo a través de una encuesta por muestreo estadístico en el primer semestre de 2019, en la que se consideró a los productores que gestionaban sistemas de producción bovina en el área de estudio. Para tal fin, se realizaron visitas de campo a las unidades de producción y se aplicó una cédula de entrevista semiestructurada; se verificó la información proporcionada mediante observación directa.

Entrevista. Con este procedimiento se obtuvo información sobre el manejo animal y la gestión de los sistemas de producción bovina de interés. La cédula de entrevista comprendió 134 preguntas, distribuidas en las siguientes secciones: a) datos generales, b) inventario animal, c) alimentación, d) reproducción, e) genética, f) sanidad, g) manejo de praderas, $h$ ) rutinas de manejo, i) asistencia técnica, j) recursos naturales, k) comercialización y I) información complementaria. La cédula de entrevista fue validada de manera preliminar en 10 unidades de producción bovina. 
Muestreo. Debido a la naturaleza exploratoria de este estudio, se eligió como método de muestreo el de tipo irrestricto aleatorio para estimación de proporciones, para lo cual se consideró una misma probabilidad a priori (50\%) de selección del sistema de producción según su finalidad, de tipo carne o doble propósito. Por otra parte, se consideró como marco muestral el listado de productores consolidado y como unidad de producción de interés al predio con inventario bovino igual o mayor a 30 cabezas. El error de estimación fue de $10 \%$ y con éste el tamaño de la muestra final fue de 33 productores, los cuales se seleccionaron completamente al azar del listado previamente consolidado. Los criterios para determinar el tamaño de la muestra final se aprecian en la siguiente tabla.

Tabla 1. Criterios estadísticos para el tamaño de la muestra.

\begin{tabular}{l}
\hline \multicolumn{1}{c}{ Descripción del criterio Fórmula matemática } \\
$\mathrm{n}$ : Tamaño de la muestra \\
$\mathrm{N}$ : Número total de productores \\
enlistados $=49$ \\
$\mathrm{p}:$ Probabilidad finalidad productiva \\
vocación cárnica \\
q: Probabilidad finalidad productiva \\
doble propósito \\
$\begin{array}{l}\mathrm{D}: \text { Parámetro de muestreo } \\
\mathrm{B}: \text { Error de estimación }(0.10) \\
\mathrm{n}=33.1\end{array}$
\end{tabular}

Sistematización y análisis de los datos. Mediante el uso de una hoja de cálculo Excel Microsoft Office 2019 se registró la información obtenida en campo y con ésta se conformó una base de datos con las variables de interés del estudio. Una vez validada la información, se emplearon procedimientos de estadística descriptiva para estimar los parámetros de desempeño productivo de los hatos.

Por su parte, en el procedimiento para la estimación de los costos de producción e ingresos, se consideraron los valores anualizados de adquisición de insumos, mano de obra, depreciación de activos, otros gastos y de las ventas. La rentabilidad del sistema de producción, se determinó al restar el costo total de producción al valor de las ventas; la relación entre estos valores proporcionó el índice de rentabilidad respectivo. Con el fin de facilitar la compresión de los resultados, se presentan los valores monetarios de los parámetros estimados en dólares americanos (USD), se empleó la tasa de cambio representativa del mercado del Banco de la República de Colombia y que correspondió a \$3.942 del día 14 de mayo de 2020. Asimismo, y con el fin de homogenizar los parámetros se presentan los valores promedio anualizados por vientre y unidad de superficie según el tipo de sistema de producción identificado en el área de estudio.

Determinación del nivel tecnológico. LoS criterios y parámetros empleados para definir el nivel tecnológico (NT) de los sistemas de producción bovina considerados en este estudio, fueron los propuestos por la Federación Colombiana de Ganaderos en 2012, los que se presentan en la siguiente tabla.

Tabla 2. Parámetros de clasificación por nivel tecnológico de unidades de producción bovina en Colombia.

\begin{tabular}{llcc}
\hline \multirow{2}{*}{ Parámetro de clasificación } & \multicolumn{3}{c}{ Nivel de tecnología } \\
\cline { 2 - 3 } & Bajo & Medio & Alto \\
\hline
\end{tabular}

1. Dispone de sistema de riego

2. Dispone de pastos mejorados.

3. Se suministra suplementos alimenticios.

4. Se realizan planes de mejora genética

Al menos debe presentar un solo parámetro

5. Se realiza mecanización de las praderas.

6. Se realiza rotación programada de potreros.

7.Se emplean registros técnicos, contables y económicos. 
Parra-Cortés y Magaña-Magaña - Sistemas de producción bovina en trópico bajo colombiano

\section{RESULTADOS}

Descripción del predio. Los predios destinados a la producción bovina cuentan con una superficie promedio de 318.2 hectáreas, el rango de ésta fue de 27 a 1.760. La distribución de la superficie fue $63.5 \%$ de praderas en su mayoría del género Brachiaria y Panincum, $1.6 \%$ de sistema de silvopastoreo (Gliricidia, Guazuma, Leucaena y Crescentia), $14.6 \%$ de reserva forestal y el restante correspondió a construcciones, cultivos agrícolas diversos y reservorios de agua. Por su parte, se determinó que la tenencia de la tierra de las unidades de producción fue $87 \%$ de propiedad privada y $13 \%$ en arrendamiento.

Características socioeconómicas de los productores. Se evidenció que las unidades de producción fueron gestionadas en $96 \%$ por hombres. Entre las principales características socioeconómicas de los productores se destaca la edad promedio de 58 años, una escolaridad de nivel superior (16 años), así como experiencia en la actividad ganadera de 20 años. En el 52\% de los casos, se determinó que los productores ejercían la ganadería como principal actividad económica, condición relacionada al nivel de escolaridad, ya que al poseer un mayor nivel educativo logran integrarse al mercado laboral de manera formal $y$, a su vez, el $48 \%$ practican la ganadería como actividad complementaria destinada al ahorro y generación de ingresos adicionales.

Finalidad productiva. El ganado de vocación cárnica constituyó sistemas de producción con finalidades de: a) Pie de cría, b) Ciclo completo y c) Cría y levante, así como combinaciones entre éstas. Además, se identificaron en el área de estudio unidades de producción dedicadas a sistema de Doble propósito y Pie de cría, (Tabla 3). La elección de la finalidad productiva se relaciona con el mercado de productos predominante en el área geográfica donde se localiza cada predio ganadero. Sin embargo, en la actualidad la raza Hartón del Valle se encuentra en expansión por la Costa atlántica y Ios Llanos Orientales de Colombia. Por lo tanto, productores de esta raza, además de la venta de leche y terneros, se esfuerzan por satisfacer la demanda de animales de Pie de cría con supuesto valor genético para la mejora de los hatos.
Tabla 3. Distribución de hatos según finalidad productiva y raza.

\begin{tabular}{lccc}
\hline \multirow{2}{*}{ Finalidad productiva } & FH & \multicolumn{2}{c}{ FTR(\%) } \\
\cline { 2 - 4 } & $\mathbf{( \% )}$ & RO & HV \\
\hline Carne-Pie de cría & 17 & 100 & - \\
Carne-ciclo completo y Pie de cría & 17 & 75 & 25 \\
Carne-ciclo completo & 13 & 100 & - \\
Carne-cría y levante & 17 & 75 & 25 \\
Doble propósito-Pie de cría & 22 & 100 & - \\
Doble propósito & 14 & 100 & - \\
\hline
\end{tabular}

$\mathrm{FH}=$ Frecuencia de hatos; FTR(\%)= Frecuencia por tipo racial $(\%) ; \mathrm{RO}=$ Romosinuano; HV= Hartón del Valle.

Tamaño y estructura de hato. El inventario animal de las unidades de producción varió de 19 a 971 cabezas, con una media de 349 y desviación estándar de 289 cabezas. El inventario total de la muestra fue de 8.029 cabezas, con una estructura del hato conformada por sementales $(2 \%)$, vacas $(45 \%)$, novillas de levante y vientre $(17 \%)$, machos en desarrollo y toretes $(10 \%)$, Terneras $(14 \%)$ y Terneros (12\%). Por otra parte, y con relación al tipo racial, en el $57 \%$ de los sistemas de producción se observaron ejemplares de la raza Romosinuano y en $43 \%$ la raza Hartón del Valle.

Descripción del manejo zootécnico. El 92\% de los productores de la muestra gestionaron sus hatos en sistemas de libre pastoreo, mientras el restante lo hicieron en semi-estabulación. Con respecto al complemento alimenticio del hato, se destaca el suministro de alimento balanceado a crías (13\%) y animales adultos ( $9 \%)$, no se reportó esta práctica para otros ejemplares. La totalidad de los productores ofrecieron ad libitum sal mineralizada de tipo comercial a todo el hato. En cuanto al manejo de la reproducción animal, en el $96 \%$ de los sistemas de producción se realizó la observación de celos, así como en igual proporción el método de cubrición de las hembras fue monta continua. Por su parte, en la muestra predominó la implementación de programas de mejoramiento genético mediante la introducción a los hatos de toros criollos, reconocidos como puros y con registro genealógico emitido por una asociación de criadores. 
El manejo sanitario del hato consistió en programas de control preventivo que incluyó la aplicación de vacunas para enfermedades de control oficial en Colombia (Aftosa, Rabia paralítica y Brucelosis); así como vacunas para enfermedades clostridiales. Asimismo, el $70 \%$ de los productores realizó aspersiones con productos químicos para el control de ectoparásitos (moscas y garrapatas) y el suministro de fármacos orales e inyectables para contrarrestar las afectaciones por endoparásitos. Los productores de la muestra

\section{A}

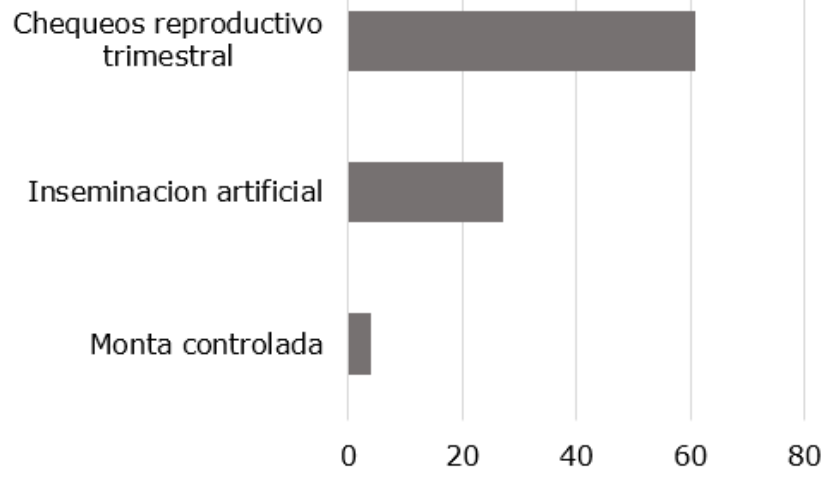

\section{c}

Asistencia veterinaria permanente

Farmacos orales para
endoparasitos

Control biológico de ectoparasitos

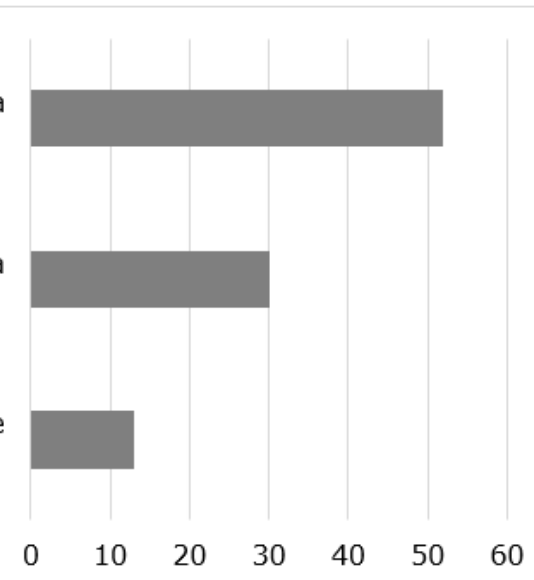

manifestaron que, en el último año, las pérdidas por problemas sanitarios no sobrepasaron el $3 \%$ del inventario animal.

Finalmente, se señala que la distribución de los sistemas de producción, según la forma de pastoreo correspondió a $52 \%$ el de tipo racional intensivo (pastoreo racional Voisin y pastoreo de ultra alta densidad), $44 \%$ de tipo rotacional y el restante al silvopastoreo. Otras prácticas de manejo del hato se presentan en la Figura 2.

\section{B}

Suministro de ensilaje

Suministro de heno

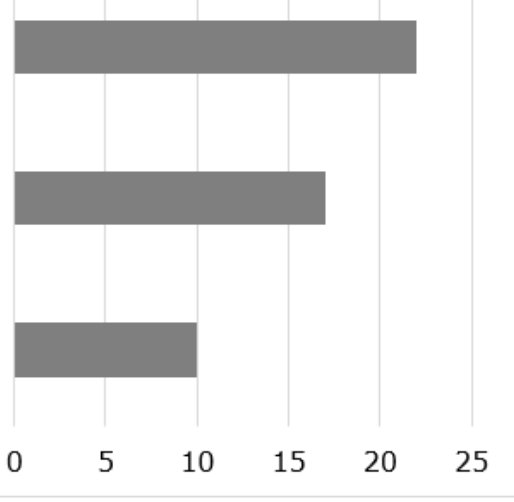

D

Pasturas mejoradas

Fertilización con abonos químicos

Pastoreo rotacional

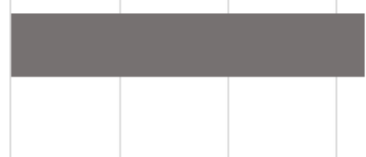
forministro de sal
formulada

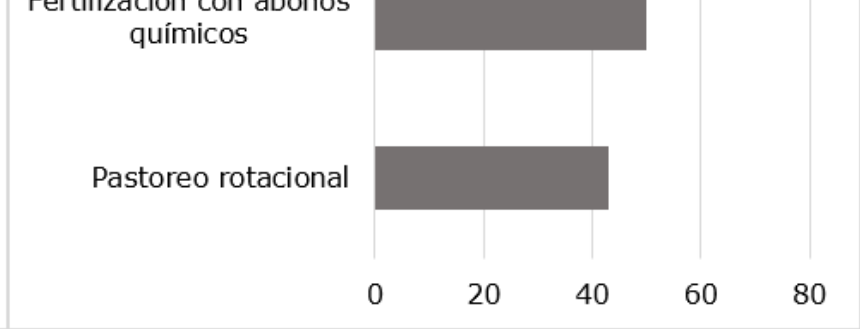

Figura 2. Frecuencia de adopción de prácticas de manejo animal y de praderas en la muestra. A. Prácticas de manejo reproductivo, B. Prácticas de manejo nutricional, C. Prácticas de manejo sanitario, D. Prácticas de manejo de praderas.

Parámetros productivos. Los principales parámetros de desempeño animal para la raza Romosinuano fueron peso al nacimiento $y$ al destete de $28.04 \pm 1.3 \mathrm{~kg}$ y $185.04 \pm 45.48$ $\mathrm{kg}$, así como fertilidad de $80 \pm 16 \%$ e intervalo entre partos de $408 \pm 43$ días. Al respecto, para la raza Hartón del Valle se observó $28.10 \pm$ $0.32 \mathrm{~kg}$ y $199.5 \pm 8.32 \mathrm{~kg}$, y de $82 \pm 9 \%$ y 354 \pm 13 días, respectivamente. Asimismo, en la muestra se determinó un índice de pureza racial de 0.96 y 0.68 para machos reproductores y vientres, respectivamente. Por otra parte, se reportó para sistemas de Doble propósito una producción diaria de leche de $4.81 \pm 0.92$ litros por vaca y una duración de la lactancia de $258.75 \pm 15.53$ días.

Características administrativas. LoS productores de la muestra manifestaron que ejercen una visión empresarial, condición que 
se vinculó al tamaño del predio, inventario del hato y nivel de escolaridad. Por su parte, se evidenció el registro permanente de datos productivos para todos los hatos. No obstante, en solo el $52 \%$ de los casos se reportó el registro de datos contables y económicos, esto mediante anotaciones de campo y softwares especializados. Asimismo, se determinó que la mano de obra en las unidades de producción correspondió a trabajadores permanentes por contrato formal y pago mensual, así como trabajadores ocasionales por contrato informal y pago por jornal; estas características son propias de la ganadería de tipo empresarial en zonas tropicales de Colombia. Finalmente, se destaca que la asistencia técnica consistió, por lo general, en recomendaciones sobre manejo reproductivo del hato, brindada por médicos veterinarios calificados.

Nivel tecnológico. Con base en los criterios de FEDEGAN, se evidenció que el $48 \%$ de las unidades de producción presentan nivel tecnológico medio y el $52 \%$ fue de nivel alto; asimismo, no se encontró en la muestra unidades con baja tecnología. Es importante mencionar que el nivel tecnológico observado se relaciona de forma directa con las características socioeconómicas del productor, como el nivel de escolaridad, años de experiencia en la actividad y la capacidad de inversión. Los principales indicadores de desempeño animal, según el nivel tecnológico y tipo racial, se presentan en la Tabla 4.

Tabla 4. Parámetros de desempeño animal, según tipo racial y nivel tecnológico.

\begin{tabular}{|c|c|c|c|c|}
\hline \multirow{2}{*}{ Variable } & \multicolumn{2}{|c|}{ Romosinuano } & \multicolumn{2}{|c|}{ Hartón del Valle } \\
\hline & NTM & NTA & NTM & NTA \\
\hline Tasa de fertilidad anual (\%) & $77.0 \pm 16.0$ & $82.6 \pm 17.1$ & $82.0 \pm 13.0$ & $81.20 \pm 5.2$ \\
\hline Intervalo entre parto (días) & $380.8 \pm 50.0$ & $431.6 \pm 30.0$ & 360.0 & $348.0 \pm 16.4$ \\
\hline Tasa de natalidad (\%) & $90.0 \pm 10.5$ & $86.5 \pm 9.0$ & $82.0 \pm 4.5$ & $83.50 \pm 8.9$ \\
\hline Días abiertos & $102.5 \pm 40.5$ & $151.7 \pm 13.3$ & $72.0 \pm 16.4$ & $90.60 \pm 19.5$ \\
\hline Edad 1.er servicio nov. (meses) & $26.7 \pm 6.0$ & $22.6 \pm 4.0$ & $25.40 \pm 1.9$ & $26.80 \pm 1.3$ \\
\hline Peso 1.er servicio nov. (kg) & $355.0 \pm 70.0$ & $310 \pm 19.1$ & $312.0 \pm 10.9$ & $314.0 \pm 19.5$ \\
\hline Edad 1.er parto (meses) & $35.7 \pm 6.0$ & $31.6 \pm 4.0$ & $34.40 \pm 1.9$ & $35.80 \pm 1.3$ \\
\hline Peso nacimiento $(\mathrm{kg})$ & $27.8 \pm 2.0$ & $28.2 \pm 1.1$ & 28.0 & $28.20 \pm 0.4$ \\
\hline Peso destete $(\mathrm{kg})$ & $170.0 \pm 24.0$ & $197.9 \pm 57.0$ & 200.0 & $199.0 \pm 12.4$ \\
\hline Edad destete (días) & $245.0 \pm 12.2$ & $242.1 \pm 28.0$ & 240.0 & 240.0 \\
\hline
\end{tabular}

NTM: Nivel tecnologico medio; NTA: Nivel tecnológico alto.

Precios de venta. En el área de estudio los precios de venta de los principales productos comercializados fueron de $0.32,1.06$ y 954.05 USD para el litro de leche, kilogramo de animal en pie para abasto (ternero desteto y engordado) y ejemplar de pie de cría, respectivamente. El precio de los dos primeros productos se determina por el comportamiento del mercado regional y nacional, mientras que en el caso del tercer producto su precio lo determina el productor $y$, en menor frecuencia, se fija por acuerdo mutuo entre el comprador y vendedor.

Indicadores de desempeño económico. El costo total de producción por unidad ganadera en la muestra, se integró por el $46 \%$ de los costos de tipo variable y la parte restante lo constituyó el valor de los costos fijos. Dentro del primer tipo de costo sobresalen los rubros de pago de mano de obra (21.3\%) y la compra de suplementos para la alimentación animal (70.4\%). Por otra parte, se determinó que el ingreso promedio por ventas fue de 73.230 USD en 2019, lo que favoreció un rango de rentabilidad de 3.235 a 37.198 USD. La estructura de costos y los indicadores de desempeño económico, según finalidad de las unidades de producción, se presenta en la siguiente tabla. 
Parra-Cortés y Magaña-Magaña - Sistemas de producción bovina en trópico bajo colombiano

Tabla 5. Indicadores de desempeño económico de los sistemas bovinos según finalidad productiva.

\begin{tabular}{|c|c|c|c|c|c|c|c|c|c|c|c|c|}
\hline \multirow{2}{*}{ Indicador } & \multicolumn{2}{|c|}{ Carne genética } & \multicolumn{2}{|c|}{$\begin{array}{c}\text { Carne CC y } \\
\text { genética }\end{array}$} & \multicolumn{2}{|c|}{ Carne CC } & \multicolumn{2}{|c|}{ Carne CL } & \multicolumn{2}{|c|}{ DP y genética } & \multicolumn{2}{|c|}{ DP } \\
\hline & $\boldsymbol{\mu}$ & $\boldsymbol{\sigma}$ & $\boldsymbol{\mu}$ & $\boldsymbol{\sigma}$ & $\boldsymbol{\mu}$ & $\boldsymbol{\sigma}$ & $\boldsymbol{\mu}$ & $\boldsymbol{\sigma}$ & $\boldsymbol{\mu}$ & $\boldsymbol{\sigma}$ & $\boldsymbol{\mu}$ & $\boldsymbol{\sigma}$ \\
\hline Hectáreas en pastoreo & 33.7 & 18.4 & 219.5 & 282.8 & 217.0 & 175.1 & 424.5 & 333.0 & 179.8 & 136.6 & 198.0 & 27.1 \\
\hline Número de vientres & 30.0 & 16.0 & 110.0 & 92.0 & 250.0 & 96.0 & 422.0 & 135.0 & 142.0 & 115.0 & 251.0 & 103.0 \\
\hline Ingreso total & 22.421 & 4.448 & 48.362 & 36.224 & 71.328 & 27.406 & 106.352 & 56.037 & 45.350 & 25.420 & 185.477 & 5.493 \\
\hline Costo variable & 7.736 & 2.193 & 9.808 & 3.494 & 17.228 & 3.271 & 19.146 & 4.891 & 9.730 & 4.740 & 126.516 & 22.672 \\
\hline Mano de obra & 5.009 & 1.445 & 5.391 & 1.743 & 7.477 & 1.624 & 6.540 & 2.297 & 4.730 & 1.666 & 6.540 & - \\
\hline Alimentación & 1.560 & 1.414 & 2.309 & 1.306 & 6.794 & 1.296 & 9.473 & 4.120 & 2.965 & 2.260 & 117.558 & 22.580 \\
\hline Medicamentos & 94.0 & 63.0 & 326.0 & 226.0 & 726.0 & 218.0 & 800.0 & 407.0 & 257.0 & 182.0 & 472.0 & 70.0 \\
\hline Chequeo reprod. & 75.0 & 55.0 & 292.0 & 305.0 & 328.0 & 193.0 & 843.0 & 537.0 & 433.0 & 349.0 & 763.0 & 313.0 \\
\hline Mantenimiento & 793.0 & 317.0 & 1.015 & 293.0 & 1.268 & - & 1.110 & 317.0 & 888.0 & 347.0 & 1.226 & 73.0 \\
\hline Combustibles & 872.0 & 159.0 & 1.364 & 476.0 & 1.776 & - & 1.364 & 476.0 & 1.218 & 526.0 & 1.057 & 183.0 \\
\hline Servicios públicos & 127.0 & - & 127.0 & - & 127.0 & - & 127.0 & - & 127.0 & - & 127.0 & - \\
\hline Costo Fijos & 9.820 & 2.441 & 26.324 & 27.517 & 36.055 & 17.559 & 74.438 & 58.053 & 21.058 & 12.164 & 26.898 & 944.0 \\
\hline Otros pagos fijos & 76.0 & - & 76.0 & - & 76.0 & - & 76.0 & - & 76.0 & - & 76.0 & - \\
\hline Depreciación & 8.278 & 2.412 & 22.413 & 27.790 & 32.570 & 16.738 & 70.061 & 56.201 & 16.152 & 13.632 & 13.781 & 11.583 \\
\hline Impuestos & 673.0 & 133.0 & 1.451 & 1.087 & 2.140 & 822.0 & 3.191 & 1.681 & 1.361 & 763.0 & 5.564 & 165.0 \\
\hline Arrendamiento & - & - & 1.370 & 2.740 & - & - & - & - & 2.581 & 5.772 & 6.251 & 10.826 \\
\hline Costo total & 17.556 & 3.964 & 36.132 & 29.533 & 53.282 & 19.459 & 93.584 & 60.464 & 30.787 & 16.589 & 153.414 & 22.345 \\
\hline Rentabilidad & 4.865 & 1.905 & 12.230 & 7.525 & 18.046 & 9.252 & 12.768 & 7.050 & 14.563 & 9.208 & 32.063 & 20.655 \\
\hline Índice de rentabilidad & $29.0 \%$ & $13.0 \%$ & $37.0 \%$ & $15.0 \%$ & $33.0 \%$ & $9.0 \%$ & $21.0 \%$ & $16.0 \%$ & $45.0 \%$ & $12.0 \%$ & $23.0 \%$ & $18.0 \%$ \\
\hline Rentabilidad ha/año & 144.0 & 103.0 & 56.0 & 27.0 & 83.0 & 53.0 & 30.0 & 21.0 & 81.0 & 67.0 & 162.0 & 763.0 \\
\hline Rentabilidad vientre/año & 164.0 & 121.0 & 111.0 & 82.0 & 72.0 & 96.0 & 30.0 & 52.0 & 102.0 & 80.0 & 128.0 & 201.0 \\
\hline
\end{tabular}

$\mathrm{CC}=$ Ciclo completo; $\mathrm{CL}=$ Cría y Levante; $\mathrm{DP}=$ Doble propósito.

Todos valores económicos están expresados en dólares (USD). Tasa de cambio del 14 de mayo de 2020, publicada por el Banco de la Republica de Colombia

\section{DISCUSIÓN}

A partir del desembarque de los primeros ganados en América durante La Conquista, se conformaron grupos de razas locales conocidos como "criollos", los cuales integraron la totalidad de la ganadería colombiana hasta inicios del siglo XX (11). No obstante, en tiempos modernos la introducción de razas foráneas y los cruzamientos indiscriminados ocasionaron la reducción drástica de los núcleos de bovinos criollos en países de América Latina (3). Durante la segunda mitad del siglo XX, en Colombia diversos autores publicaron resultados sobre el desempeño productivo de los hatos conformados por ejemplares de las diferentes razas criollas que existen en el país (8). Sin embargo, se desconocen las cifras oficiales del inventario y características actuales de los sistemas constituidos por bovinos criollos colombianos. Por lo tanto, en esta discusión, se consideraron antecedentes nacionales de las últimas dos décadas, así como resultados recientes de trabajos similares realizados en Mexico.

Hacia el 2002, los ejemplares de razas criollas solo se vincularon a sistemas de producción de tipo extensivo, caracterizado por el agotamiento de los recursos naturales, escasa inversión de capital y bajo nivel tecnológico (12). Por el contrario, en la actualidad este trabajo asoció a los bovinos criollos con sistemas de producción de tipo empresarial; las evidencias obtenidas indican que existe una relación directa entre el tamaño del predio y el alcance de la visión empresarial. Respecto al tamaño del predio, se observó en México una superficie promedio 
de 110 hectáreas en sistemas ganaderos caracterizados por su manejo empresarial. El tamaño del predio en la muestra fue superior al reportado para otras ganaderías, como las del Departamento del Tolima (13) y de Los Llanos Orientales de Colombia (14). Por su parte, se reportó para sistemas de doble propósito en México un inventario de 300 animales, gestionados bajo un nivel tecnológico especializado; condición similar fue observada en la muestra de este estudio.

El censo de razas criollas colombianas publicado en 1999, reportó una edad promedio del productor de 48 años y un nivel de educación superior para el 36.9\% (8). La edad del productor en la muestra superó en diez años al promedio censal y la proporción con educación superior fue mayor. En México, la edad de los productores y su experiencia en ganadería, fueron similares a los valores obtenidos en la muestra $(15,16,17,18)$. La edad avanzada de los productores se asocia a procesos de herencia de la tierra en el medio rural. Asimismo, en México se observó que sistemas de producción bovina semi-tecnificados y tecnificados fueron gestionados por productores con nivel de educación superior. En el presente, se señala que los bovinos criollos colombianos son parte de agroecosistemas con nivel tecnológico sobresaliente, gestionados por productores con edad avanzada y escolaridad de nivel superior.

En cuanto al desempeño animal, en 2018 se determinó para ejemplares Romosinuano un peso al destete de $172 \mathrm{~kg}$ a los 219 días, esto en condiciones del trópico mexicano (19). Los valores fueron similares en la muestra, pero superaron lo estimado para esta misma raza en hatos ubicados en los estados mexicanos de Tabasco y Veracruz (20). Con relación a la raza Hartón del Valle, en 2001 se reportó en Colombia un rango de peso al nacimiento de 31 a $35 \mathrm{~kg}$ y al destete de 165 a $178 \mathrm{~kg}$ a los 245 días (21). Para esta última raza, se determinó en la muestra un peso inferior al nacimiento, pero superior al destete. En términos generales, se considera que el peso al nacimiento superior a $30 \mathrm{~kg}$, como ocurre en razas foráneas, se relaciona con problemas de distocia al momento del parto. Por lo tanto, el nacimiento de crías livianas es una característica deseable propia de los bovinos criollos colombianos (22).

Respecto al desempeño reproductivo, para la raza Hartón del Valle se determinó un rango de intervalo entre partos de 360 a 390 días (8), este parámetro fue similar en la muestra. Por su parte, para hatos en el trópico bajo colombiano, se reportó para la raza Romosinuano un intervalo entre parto de $422 \pm 131$ días (23), valor similar al de la muestra. Recientemente en zonas tropicales de México, se observó que los parámetros de edad y peso al primer servicio de novillas Romosinuano, disminuían al progresar el nivel tecnológico del sistema de producción (24), condición que también se observó en este estudio. Por su parte, se considera que la mejora en las prácticas de alimentación, se favorece el incremento del peso al primer parto y la recuperación postparto de las hembras criollas (25).

La producción lechera de la raza Hartón del Valle se estimó en 1.683 litros y 284 días, es decir 5.9 litros/vaca/día, en condiciones del trópico medio colombiano (8); estos parámetros fueron menores en la muestra. No obstante, en Los Llanos Orientales, se reportó para esta misma raza un rango de producción de leche de 4.0 a 7.0 litros/vaca/día $(26,27)$. La producción diaria de leche está relacionada con la calidad de la alimentación y las condiciones de confort de los animales. El trópico bajo colombiano presenta una oferta limitada de forrajes e índices de calor extremo; por lo tanto, se reportó una menor producción láctea en los hatos de interés. Se observó entre los productores entrevistados una preferencia por los métodos de pastoreo racional intensivo, característica que contrastó con los reportes del siglo $X X$, donde se señaló al pastoreo continuo como el más común. La rusticidad de los ganados criollos favorece que los productores realicen un menor suministro de balanceados, en el caso de la oferta de sal mineralizada se observó que fue constante, como práctica común entre los productores para complementar los nutrientes provenientes de los forrajes consumidos por los ganados.

Con relación al desempeño económico de los hatos, se observaron mayores gastos en alimentación y pago de mano de obra, en los sistemas de Doble propósito y Cría y levante. Además, se destaca que el índice de rentabilidad de los sistemas de producción, superó el valor mínimo aceptable, este definido en 12\% anual (28). Los resultados económicos sobresalientes se atribuyeron a la venta de ejemplares de pie de cría.

En conclusión, los atributos identificados en los sistemas de producción bovina basados en las razas criollas Romosinuano y Hartón del Valle, se vincularon tanto a la disponibilidad de recursos naturales, capital de trabajo, características socioeconómicas de los productores, así como a su capacidad de gestión empresarial. Los cambios actuales en el entorno demandan a estos 
sistemas ganaderos respuestas tecnológicas más eficientes, condición que implica el incremento en la oferta de carne y leche a bajos costos de producción. Los resultados obtenidos posicionaron a los métodos de pastoreo racional intensivo y al aprovechamiento de las razas criollas, como alternativas idóneas para la mejora del desempeño técnico-económico de los hatos en zonas tropicales de Colombia. Asimismo, se resalta como hallazgo importante el nivel tecnológico de tipo medio-alto que se determinó para los sistemas de producción de interés; siendo más rentable el orientado al Doble propósito y Pie de cría. Las evidencias obtenidas sugieren la importancia de promover prácticas de ganadería sostenible en pastoreo que incluyan el aprovechamiento de recursos locales. Por su parte, en consonancia con los resultados obtenidos en este estudio, se recomienda multiplicar los núcleos de bovinos criollos y su cruzamiento con ejemplares de otras razas convencionales; esto con el fin de conferir genes de adaptabilidad a las crías para alcanzar mejores indicadores de productividad y rentabilidad en el sector ganadero colombiano.

\section{Conflicto de intereses}

Los autores del presente estudio declaramos que no existe conflicto de intereses con la publicación de este manuscrito.

\section{Agradecimientos}

Al comité directivo y productores que conforman la Asociación Nacional de Criadores de Razas Criollas y Colombianas (ASOCRIOLLO) y la Asociación Nacional de Criadores de Razas Criollas y Colombianas de Los Llanos Orientales (ASOCRIOLLANOS).

\section{REFERENCIAS}

1. Bava L, Sandrucci A, Zucali M, Guerci M, Tamburini A. How can farming intensification affect the environmental impact of milk production?. J Dairy Sci. 2014; 97(7):45794593. https://doi.org/10.3168/jds.2013-7530

2. Thornton P. Livestock production: recent trends, future prospects. Philos Trans R Soc Lond B Biol Sci. 2010; 365(1554): 2853-2867. https://doi.org/10.1098/rstb.2010.0134

3. Núñez-Domínguez $R$, Ramírez-Valverde $R$, Saavedra-Jiménez LA, García-Muñiz JG. La adaptabilidad de los recursos zoogenéticos Criollos, base para enfrentar los desafíos de la producción animal. Arch Zootec. 2016; 65(251):461-468. https://doi. org/10.21071/az.v65i251.717

4. Parra-Cortés RI, Magaña-Magaña MA, Piñeiro-Vázquez AT. Intensificación sostenible de la ganadería bovina tropical basada en recursos locales: alternativa de mitigación ambiental para América Latina. Revisión Bibliográfica. ITEA-Inf Tec Econ Ag. 2019; 115(4):342-359. https://doi.org/10.12706/ itea.2019.003

5. De Alba J. El libro de los bovinos criollos de América. Primera edición. México: Colegio de Postgraduados; 2011.

6. FAO-DAD-IS. Sistema de Información de Diversidad de Animales Domésticos (DADIS) [Online]. Roma: FAO; 2018. Disponible en: http://www.fao.org/dad-is
7. ICA. Censo Pecuario Nacional [Online]. Bogotá: Instituto Colombiano Agropecuario; 2019 [Accessed 2020 junio 18]. URL Disponible en: https://www.ica.gov.co/ areas/pecuaria/servicios/epidemiologiaveterinaria/censos-2016/censo-2018

8. ICA. Censo y caracterización de los sistemas de producción de ganado criollo y colombiano. Primera edición. Santafé de Bogotá, D.C: Instituto Colombiano Agropecuario; 1999..

9. MADR - ASOCRIOLLO Colombia. Razas criollas y colombianas puras: memoria Convenio 135-01. Primera edición. Santafé de Bogotá, D.C: MADR - ASOCRIOLLO Colombia; 2003.

10. IGAC. Clasificaciones climáticas de Colombia [Online]. Bogotá: IGAC; 2011 [Accessed 14 May 2020]. URL Disponible en: http://www.ideam.gov. co/documents/21021/21789/climas+\% 5BModo+de+compatibilidad\%5D.pdf/ d8c85704-a07a-4290-ba65-f2042ce99ff9

11. Lopez A, Saldarriaga O, Arango A, Rugeles M, Zuluaga F, Olivera $M$ et al. Ganado Blanco Orijenegro (BOM): Una alternativa para la producción en Colombia. Rev Col Cienc Pec. 2001; 14(2):121-128. https:// revistas.udea.edu.co/index.php/rccp/ article/view/323758 
12. Mahecha L, Gallego L, Peláez F. Situación actual de la ganadería de carne en Colombia y alternativas para impulsar su competitividad y sostenibilidad. Rev Col Cienc Pec. 2002; 15(2):213-225. https:// revistas.udea.edu.co/index.php/rccp/ article/view/323816

13. Medina-Ríos E, Mora-Delgado J, RodríguezMárquez M. Indicadores bioeconómicos de fincas ganaderas del trópico bajo del norte del departamento del Tolima. Rev Tumbaga. 2016; 1(11):81-96. http:// revistas.ut.edu.co/index.php/tumbaga/ article/view/1304/992

14. Cortés H, Aguilar C, Vera R. Sistemas bovinos doble propósito en el trópico bajo de Colombia, modelo de simulación. Arch Zootec. 2003; 52(197):25-34. http:// www.uco.es/organiza/servicios/publica/ az/php/img/web/30 $10 \quad 29$ 03cortes.pdf

15. Oros V, Díaz P, Vilaboa J, Martínez J, Torres G. Caracterización por grupos tecnológicos de los hatos ganaderos doble propósito en el municipio de Las Choapas, Veracruz, México. Rev Científ. 2011; 21(1):57-63. https://produccioncientificaluz.org/index. php/cientifica/article/view/15623/15597

16. Orantes-Zebadúa $M A$, Platas-Rosado $D$, Córdova-Avalos V, De los Santos-Lara MC, Córdova-Avalos A. Caracterización de la ganadería de doble propósito en una región de Chiapas, México. Ecosist Rec Agropec. 2014; 1(1):49-58. http://era.ujat.mx/ index.php/rera/article/view/6

17. Albarrán-Portillo $B$, Rebollar-Rebollar $S$, García-Martínez A, Rojo-Rubio R, AvilésNova F, Arriaga-Jordán C. Socioeconomic and productive characterization of dualpurpose farms oriented to milk production in a subtropical region of Mexico. Trop Anim Health Prod. 2015; 47:519-523. https:// doi.org/10.1007/s11250-014-0753-8

18. Cuevas V, Loaiza A, Espinosa J, Vélez A, Montoya M. Tipología de las explotaciones ganaderas de bovinos doble propósito en Sinaloa, México. Rev Mex Cienc Pecu. 2016; 7(1):69-83. https://doi.org/10.22319/ rmcp.v7i1.4150

19. Parra-Cortés $R$, Magaña-Magaña $M$. Características técnico-económicas de los sistemas de producción bovina basados en razas criollas introducidas en México. Ecosist Rec Agropec. 2019;6(18):535-547. http://era.ujat.mx/index.php/rera/article/ view/2160
20. Ramírez R, Martínez R, Núñez R, García J. Genetic parameters and trends of growth traits for Romosinuano cattle in Mexico. Nova Scientia. 2018; 10(21):310-325. https://doi.org/10.21640/ns.v10i21.1595

21. Álvarez L. Raza Hartón del Valle (HdV). Primera edición. Santa fe de Bogotá D.C.: Instituto Colombiano Agropecuario; 2001.

22. Ossa G, Suárez M, Pérez J. Efectos del medio y la herencia sobre los pesos al nacimiento, al destete y a los 16 meses de edad en terneros de la raza criolla Romosinuano. Rev Corpoica. 2007; 8(2):81-92. https://repository.agrosavia. co/handle/20.500.12324/18249

23. Ossa G, Hinestroza D, Santana A, Reza $M$, Pérez $S$, Abuabara Y. Formación, desarrollo y caracterización fenotípica de los caracteres productivos y reproductivos del hato Romosinuano del banco de germoplasma de Colombia. Rev Corpoica. 2013; 14(2):231-243. https://doi. org/10.21930/rcta.vol14 num2 art:503

24. Severino $V$, Perezgrovas $R$, Muñoz J, Piñeiro A, Chay A. Caracterización del nivel tecnológico, edad a la pubertad y primer servicio de hembras Romosinuano en México. Acta universitaria. 2019; 29:1-9. https://doi.org/10.15174/au.2019.2477

25. Corpoica. Potencial productivo del ganado Romosinuano, BON, Cebú y sus cruzamientos en el valle del Sinú y bajo cauca antioqueño. Primera edición. Bogotá: Corporación Colombiana de Investigación Agropecuaria; 2008.

26. Cortés-Mora J, Cotes-Torres A, CotesTorres J. Avances en clasificación de sistemas de producción con bovinos doble propósito en Colombia. Arch Zootec. 2014; 63(243):559-562. https://www. uco.es/ucopress/az/index.php/az/article/ view/537

17. Onofre G, Parra J, Martínez R, Cassalett $\mathrm{E}$, Velásquez $\mathrm{H}$. Potencial productivo $\mathrm{y}$ calidad de la leche de razas criollas Blanco orejinegro, Hartón del Valle y Sanmartinero en piedemonte colombiano. AICA. 2015; 5:1517. http://agris.fao.org/agris-search/search. do?recordID $=\mathrm{ES} 2015104912$

28. Ramírez J, Rebollar-Rebollar A, Rebollar-Rebollar S, Jaramillo B, González F. Estudio de viabilidad económica para una engorda de bovinos en corral en el sur del estado de México. Rev Mex Agronegocios 2017; 41:742-753. http:// ri.uaemex.mx/handle/20.500.11799/68460 